\title{
Comparación de adenosina deaminasa y detección de anticuerpos anti-antígeno A60 para el diagnóstico de meningitis tuberculosa
}

\author{
Patricia García, Laura Bahamondes, Paula Reyes, Juan C. Román, Haydé Poblete y M. Elvira Balcells
}

\section{A comparative study for adenosine deaminase and anti-antigen A-60 antibodies detection for the diagnosis of tuberculous meningitis}

Background: Diagnosis of tuberculous meningitis (TBM) is hampered by the lack of rapid and accurate diagnostic tools. We evaluated the immunological response to Mycobacterium tuberculosis anti-A60 antibodies in cerebrospinal fluid (CSF) in comparison to adenosine deaminase (ADA) determination, for the diagnosis of TBM. Methods: A total of 63 CSF samples were analyzed by indirect ELISA for the detection of anti- A60 IgG, IgM and IgA. These include samples from 17 patients with confirmed TBM and 46 control patients with other infections. Results: The mean individual anti-A60 IgM, IgG and IgA CSF antibody titers were significantly higher in TBM in comparison with control groups $(\mathrm{p}<0.01)$. The best discriminatory CSF antibody for confirming TBM diagnosis was IgM, with an area under the receiver operating characteristic curve of 0.928 (95\%CI 0.834-0.978), compared to 0.863 (95\% CI: $0.752-0.936)$ for ADA testing $(\mathrm{p}=\mathrm{NS})$. The sensitivity of anti- A60 IgM CSF antibody titers (cutoff $>0.06 \mathrm{U} / \mathrm{ml}$ ) was $94.1 \%$ compared to $88.2 \%$ for ADA (cutoff $>6.2 \mathrm{U} / \mathrm{ml}$ ), p $=$ NS. Both anti A60 IgM and ADA showed the same moderate specificity (80.4\%). Two cases of TBM were correctly identified by antiA60 IgM but missed by ADA. Conclusion: The ELISA test for anti-antigen A60 antibodies (IgM) is a rapid and sensitive tool for the rapid diagnosis of TBM that can be a complement to ADA determination. The specificity of both tests is still a limitation in TBM diagnosis.

Key words: Tuberculous meningitis, tuberculosis, diagnosis, mycobacterial A 60 antigen.

Palabras clave: Meningitis tuberculosa; tuberculosis; antígeno A-60.
Pontificia Universidad Católica de Chile. Facultad de Medicina Departamento de Laboratorios Clínicos (PG).

Departamento de Radiología (PR). Laboratorio de Microbiología (JCR) Departamento de Enfermedades Infecciosas (MEB). Hospital de Enfermedades Infecciosas Dr. Lucio Córdova, Santiago (LB, HP).

Conflictos de interés de los autores: ninguno Fuentes de financiamiento: Fondos Concursables del Departamento de Laboratorios Clínicos UC.

Recibido: 12 de enero de 2011 Aceptado: 3 de septiembre de 2012

Correspondencia a: María Elvira Balcells Marty balcells@med.puc.c

\section{Introducción}

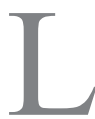

a meningitis tuberculosa (MTBC) es una enfermedad aún prevalente en países en desarrollo; sin embargo, en las últimas décadas su incidencia también se ha incrementado en países industrializados en relación a la epidemia de $\mathrm{VIH}^{1}$. Tiene una alta morbi-mortalidad que sólo puede ser evitada mediante el rápido inicio de tratamiento correcto ${ }^{2}$. No obstante, con frecuencia la administración de terapias antituberculosas es tardía debido a que la forma de presentación clínica y las alteraciones del LCR son inespecíficas, sumado a que no existen tests diagnósticos rápidos suficientemente sensibles y específicos ${ }^{3}$. La baciloscopia tiene baja sensibilidad en LCR y el cultivo de micobacterias es muy lento (30 a 60 días) y, si bien la determinación de adenosina deaminasa (ADA) es una herramienta de uso común en el proceso diagnóstico de la MTBC, también presenta resultados variables de sensibilidad y especificidad (rango 58-83\% y 71-95\%, respectivamente, en diferentes estudios $)^{4,5}$. Por otra parte, los nuevos tests diagnósticos basados en amplificación de ácidos nucleicos (RPC) para M. tuberculosis en LCR, además de ser de mayor costo y complejidad técnica, también están limitados por una baja y variable sensibilidad (50-60\%), por lo que tampoco permiten excluir una meningitis con seguridad ${ }^{6}$.

De acuerdo a varias publicaciones, la respuesta inflamatoria de M. tuberculosis en el sistema nervioso central se caracteriza por síntesis intratecal de inmunoglobulinas (Ig) oligoclonales detectables en LCR durante la infección meníngea $^{7,8}$. Numerosos componentes antigénicos de las micobacterias tal como ESAT-6, 14 kDA, MPT63, 19kDA, MPT64, 38 kDA y A60 han sido caracterizados para detección mediante técnicas de inmunoensayo y serología en el diagnóstico de tuberculosis pulmonar y extra-pulmonar-11. El rango de detección en LCR para diferentes antígenos de $M$. tuberculosis en pacientes cursando MTBC varía de 60 a 100\% en las distintas series, con importantes diferencias entre los casos individuales, técnicas de detección de anticuerpos únicos o múltiples, momento de toma de muestra en el curso de la enfermedad, status VIH del paciente y método de definición del estándar de oro (diagnóstico clínico versus cultivo) ${ }^{12-15}$.

El complejo A60 representa una fracción importante del total de antígenos de $M$. tuberculosis y la mayoría de los anticuerpos circulantes en sangre y LCR de pacientes cursando tuberculosis están dirigidos contra $A 60^{16}$. Sin embargo, el rendimiento diagnóstico de los anticuerpos 
anti-A60 en la MTBC ha sido variable en los estudios publicados ${ }^{8,17-19}$. La sensibilidad reportada ha sido inconsistente, con valores desde 50\% para detección de IgG e IgA anti-A60 en pacientes inmunocompetentes hasta $100 \%$ de detección para IgG, aun en estadios precoces de la enfermedad ${ }^{20,21}$.

En ese contexto, el objetivo del presente estudio fue determinar el rendimiento diagnóstico de tres clases de anticuerpos anti-A60 (IgG, IgM e IgA) en el diagnóstico precoz de la MTBC, y evaluar si pudiesen constituir un aporte al tradicional uso de ADA en LCR.

\section{Métodos}

Selección de muestras: Este estudio se realizó en forma retrospectiva analizando las muestras de LCR que hubiesen sido obtenidas durante el proceso diagnóstico de rutina en pacientes adultos ingresados por fiebre y con sospecha clínica de meningitis infecciosa entre noviembre de 2002 y diciembre de 2004 en el Hospital de Enfermedades Infecciosas Dr. Lucio Córdova y Hospital Clínico de la Pontificia Universidad Católica, en Santiago, Chile. De éstas, se excluyeron las muestras de LCR en que se hubiese confirmado meningitis bacteriana aguda por tinción de Gram o cultivo bacteriano positivo.

Análisis de LCR: El LCR fue estudiado mediante análisis citoquímico, recuento diferencial de leucocitos, ADA, tinción de Gram y cultivo corriente, tinción de tinta china, cultivo de hongos, tinción de Ziehl Neelsen, cultivo para $M$. tuberculosis, RPC para $M$. tuberculosis (Roche, Amplicor $\left.{ }^{\circledR}\right)$, RCR para virus herpes simplex 1 y 2 (Roche, Amplicor®) y VDRL. La actividad de ADA en LCR se determinó a $37^{\circ} \mathrm{C}$ de acuerdo al método de Guisti y Galanti. Los resultados se expresaron en UI/mL.

Definición de caso: Los casos se definieron como aquellas muestras de LCR tomadas por sospecha clínica de meningitis infecciosa y cultivo de micobacteria o RPC para M. tuberculosis positiva en LCR. Se incluyeron tanto casos confirmados mediante cultivo como por RPC debido a que datos históricos sugieren que sólo $25 \%$-70\% de los pacientes con diagnóstico de MTBC tienen diagnóstico confirmado mediante cultivo ${ }^{22}$.

Grupo control: Se definió como aquellas muestras de LCR tomadas por sospecha clínica de meningitis infecciosa en los cuales se descartaron MTBC y meningitis bacteriana aguda (Gram y cultivo corriente negativos). Incluyeron los siguientes grupos de diagnósticos:

- Meningo-encefalitis herpética: LCR con aumento de celularidad de predominio mononuclear y RPC en LCR positiva para VHS 1 ó 2. Estudio bacteriológico negativo.
- Meningitis aséptica: LCR con aumento de celularidad de predominio mononuclear y resultados negativos en todas las tinciones, cultivos y estudio molecular.

- Meningitis criptocóccica: LCR con aumento de celularidad de predominio mononuclear y cultivo de LCR positivo para Cryptococcus neoformans. Resto del estudio bacteriológico y molecular negativo.

- Síndrome febril no meníngeo: casos en los cuales se realizó punción lumbar como parte de estudio de un síndrome febril y cefalea, en los cuales el análisis de LCR resultó normal, incluyendo los cultivos y el estudio molecular.

Test de ELISA: Las muestras de LCR fueron procesadas en el Laboratorio de Microbiología de la Pontificia Universidad Católica de Chile por un operador ciego al diagnóstico. Las muestras fueron congeladas a $-20^{\circ} \mathrm{C}$ hasta el procesamiento, de manera a disminuir la variabilidad inter-ensayo. El test de ELISA se llevó a cabo de acuerdo a las recomendaciones del fabricante (ANDA TB ${ }^{\circledR}$ Anda Biologicals, Strasbourg, France). En breve, $100 \mathrm{~mL}$ de cada muestra de LCR se diluye 1:20 y se deposita en un pocillo de microtitulación cuya superficie se encuentra recubierta por antígeno A60 purificado. Luego se incuba por 1 hora a $37^{\circ} \mathrm{C}$, tras lo cual se lava 5 veces con tampón de lavado. $100 \mathrm{~mL}$ de esta solución se añaden al conjugado correspondiente (anti IgG, anti IgM y anti IgA) y se incuba por 30 minutos a $37^{\circ} \mathrm{C}$. Posteriormente se agregan $100 \mathrm{~mL}$ de la solución sustrato y se incuba por 15 minutos exactos, protegido de la luz. La reacción es detenida con ácido sulfúrico. La lectura de absorbancia se realiza a $450 \mathrm{~nm}$ y cada valor es expresado como una densidad óptica (DO). Para cada determinación se elaboró una curva de calibración, con calibradores positivos, negativos y controles.

Análisis estadístico: Fue realizado en SPSS V10.0 y MedCalc ${ }^{\circledR}$ Software empleando T test para variables continuas y Mann-Whitney para análisis de datos noparamétricos. Los resultados se expresan como DO promedio e Intervalo de Confianza de 95\% CI. El rendimiento diagnostico del test, la selección de los puntos de corte y la comparación de ELISA con ADA fue realizada mediante análisis de curva ROC (Receiver Operating Characteristic).

\section{Resultados}

En total se recolectaron 63 muestras de LCR en el período del estudio. El grupo de MTBC confirmada incluyó 17 casos (12 confirmados por cultivo de micobacterias positivo y 5 por RPC de $M$. tuberculosis positivo en LCR). El análisis de LCR reveló en los casos de MTBC una 
glucosa promedio de 48,7 mg/dl (rango 13-100), recuento de leucocitos promedio de 144 céls $/ \mathrm{mm}^{3}$ (rango 5-380) y promedio de proteínas de 29,2 g/l (rango 1-82). El grupo control incluyó 46 casos: 21 casos de meningoencefalitis herpética, 9 casos de meningitis aséptica, 6 casos de meningitis criptocóccica y 10 casos de síndrome febril no meníngeo. El análisis de LCR del grupo control reveló una glucosa promedio de 55,2 $\mathrm{mg} / \mathrm{dl}$ (rango 0-100), recuento de leucocitos promedio de 79 céls $/ \mathrm{mm}^{3}$ (rango 0-360) y de proteínas de 15,4 g/l (rango 1-128).

El valor de ADA en LCR fue analizado para todos los grupos de pacientes. El promedio de ADA en el grupo MTBC fue de 17,42 UI/ml [95\% IC: 10,1-24,7] y de 4,07 UI/ml para el grupo control [95\% IC: 2,62-5,62], $\mathrm{p}<0,0001$. El área bajo la curva ROC para el diagnostico de MTBC mediante test de ADA fue de 0,863 (95\% IC $0,752-0,936)$, con $88,2 \%$ de sensibilidad y $80,4 \%$ de especificidad con un valor de corte de $\mathrm{ADA}>6,2$. Se obtuvieron 9 resultados falsamente positivos con este corte, incluyendo 4 casos en meningitis criptocóccica (Figura 1). Al excluir los casos de meningitis criptocóccica la especificidad de ADA subió a 94,4\% (95\% IC 81,3-99,3), manteniendo el mismo nivel de corte de $\operatorname{ADA}(>6,2)$.

Los valores promedios de DO para IgG, IgM e IgA anti-A60 en el grupo MTBC fueron significativamente superiores que en el grupo control (IgG: 0,198 versus $0,137, \mathrm{p}=0,002$; IgM: 0,187 versus $0,055, \mathrm{p}<0,0001$; IgA: 0,287 versus $0,088, \mathrm{p}<0,0001)$. Al analizar con respecto a los subgrupos de pacientes del grupo control encontramos una sobreposición significativa entre los valores promedios de las Ig anti-A60 en pacientes con meningitis criptocócica y MTBC, en particular para los valores de $\operatorname{IgG}$ (IgG promedio 0,198 versus 0,217 , respectivamente, $\mathrm{p}=\mathrm{NS}$ ). El mejor poder discriminatorio para el diagnostico de MTBC se obtuvo con IgM anti-A60, con un área bajo la curva ROC de 0,928 (95\% IC 0,834-0,978), $94,1 \%$ de sensibilidad y $80,4 \%$ de especificidad para un valor de corte $>0,06$, tal como se muestra en la Tabla 1 . Los valores de DO para IgM anti-A60 en subgrupos diagnósticos individuales están graficados en la Figura 2. Un resultado falsamente negativo para $\operatorname{IgM}$ anti-A60 se obtuvo en un paciente cursando MTBC confirmada por cultivo, con un ADA en LCR de 14.

El análisis pareado mediante curvas ROC para ambas técnicas diagnósticas en LCR (ADA e IgM anti-A60) sugiere una leve mejor rendimiento diagnóstico para IgM, aunque esto no alcanzó significancia estadística $(p=N S)$, tal como se observa en la Figura 3.

En forma secundaria analizamos sólo los casos de MTBC confirmados por cultivo $(\mathrm{n}=12)$. El valor promedio de ADA en LCR resultó de 18,83 (95\% IC 10,9-26,8). La sensibilidad de ADA mejoró a 100\% (95\% IC 73,5100) y la especificidad a $82,6 \%$ (95\% IC 68,6-92,2), con un valor de corte $>6,7$. El área bajo la curva ROC para

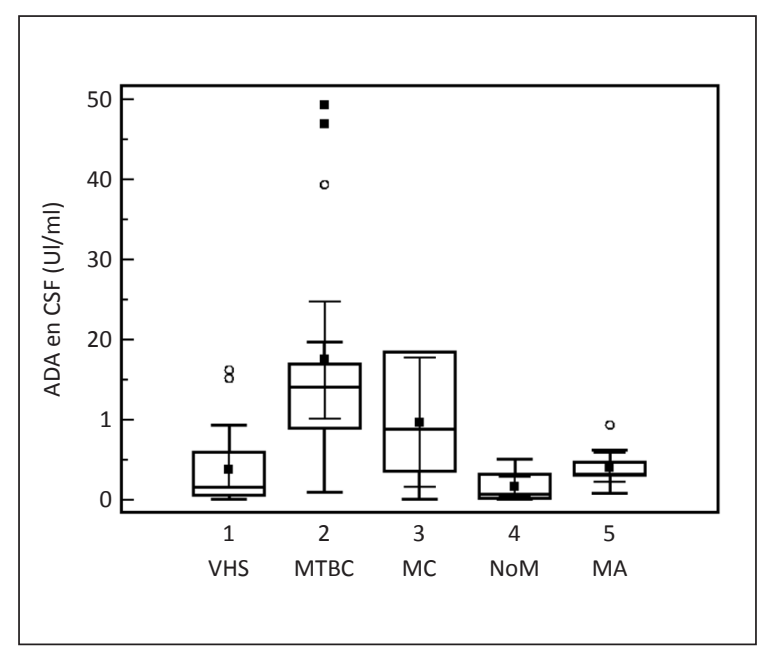

Figura 1. Valores de ADA (IU/ $\mathrm{ml}$ ) en LCR en grupo meningitis tuberculosa (MTBC) $(n=17)$ y cada uno de los subgrupos del grupo Control (meningitis herpética $(n=21)$, meningitis criptocóccica $(n=6)$, no meningitis $(n=10)$, meningitis aséptica ( $n$ $=9$ ). VHS: virus herpex simplex; MTBC: meningitis tuberculosa; MC: meningitis criptocócica; NoM: no meningitis; MA: meningitis aséptica.

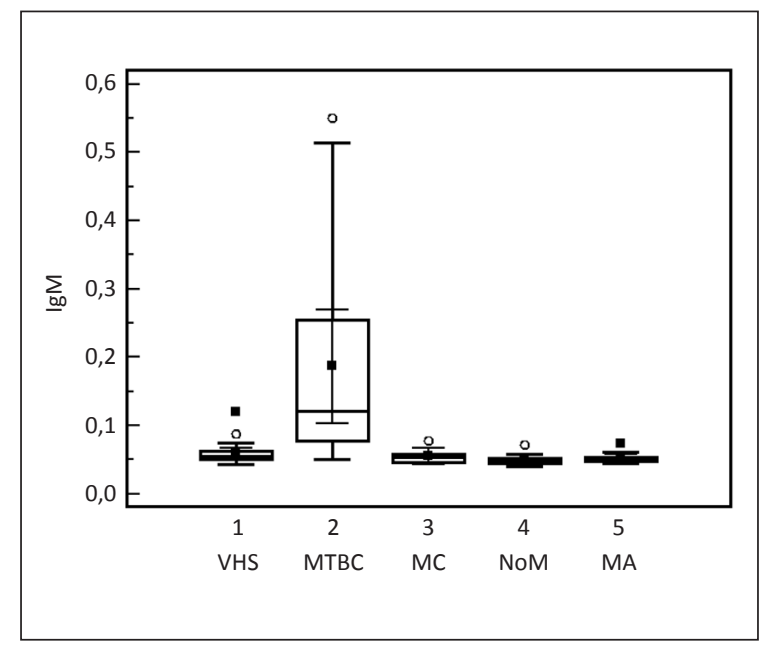

Figura 2. Valores de la densidad óptica (DO) de $\lg \mathrm{M}$ anti-A60 en el grupo meningitis tuberculosa (MTBC) $(n=17)$ y cada uno de los subgrupos del grupo Control (meningitis herpética $(n=21)$, meningitis criptocóccica $(n=6)$, no meningitis $(n=10)$, meningitis aséptica $(n=9)$. VHS: virus herpex simplex; MTBC: meningitis tuberculosa; MC: meningitis criptocócica; NoM: no meningitis; MA: meningitis aséptica.

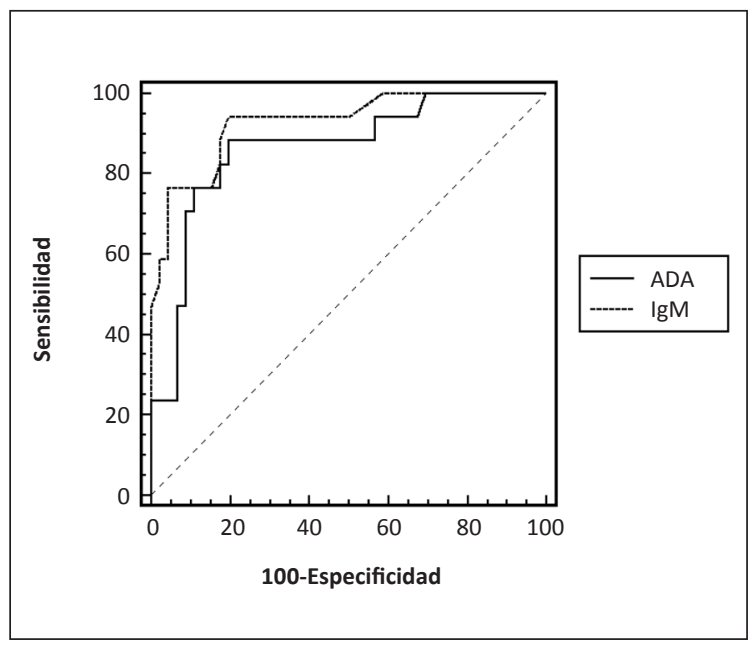

Figura 3. Análisis pareado de curvas ROC en el rendimiento diagnostico de ADA y de IgM anti-A60 en LCR en el diagnostico de la meningitis tuberculosa. 
Tabla 1. Rendimiento diagnostico de ADA y anticuerpos anti-A60 en LCR para el diagnóstico de meningitis tuberculosa

\begin{tabular}{|c|c|c|c|c|}
\hline & $\lg G$ & $\lg M$ & $\lg A$ & ADA \\
\hline Área bajo la curva ROC $(n=63)$ & $0,750[95 \% \mathrm{Cl} \quad 0,625-0,851]$ & $0,928[95 \% \mathrm{Cl} \quad 0,834-0,978]$ & $0,902[95 \% \mathrm{Cl} 0,80-0,962]$ & $0,863[95 \% \mathrm{Cl} \quad 0,752-0,936$ \\
\hline Sensibilidad & $\begin{array}{c}82,4 \%[95 \% \text { Cl } 56,6-96,2] \\
\quad(\text { corte }>0,109)\end{array}$ & $\begin{array}{c}94,1 \%[95 \% \mathrm{Cl} 71,3-99,9] \\
\text { (corte }>0,06)\end{array}$ & $\begin{array}{c}88,2 \%[95 \% \mathrm{Cl} 63,6-98,5] \\
\quad(\text { corte }>0,121)\end{array}$ & $\begin{array}{c}88,2 \%[95 \% \mathrm{Cl} \quad 63,5-98,2] \\
\text { (corte }>6,2)\end{array}$ \\
\hline Especificidad & $\begin{array}{c}65,2 \%[95 \% \mathrm{Cl} 49,8-78,6] \\
(\text { corte }>0,109)\end{array}$ & $\begin{array}{c}80,4 \%[95 \% \text { Cl } 66,1-90,6] \\
(\text { corte }>0,06)\end{array}$ & $\begin{array}{c}84,8 \%[95 \% \text { Cl } 71,1-93,7] \\
\text { (corte }>0,121)\end{array}$ & 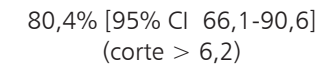 \\
\hline
\end{tabular}

ADA mejoró a 0,933, [95\% IC 0,835-0,982], p=0,001. El rendimiento diagnóstico de IgM anti-A60 no se modificó significativamente al analizar sólo los casos con cultivo positivo, con un área bajo la curva ROC en 0,929 , [95\% IC 0,83-0,98], $\mathrm{p}=0,001$.

\section{Discusión}

El presente estudio sugiere que la detección de inmunoglobulina anti-A60 puede ser una herramienta diagnóstica comparable a ADA en el diagnóstico de MTBC. El rendimiento diagnóstico de IgM anti-A60 (sensibilidad de $94,1 \%$ y especificidad de $80,4 \%$ ) resultó incluso superior a lo reportado en numerosos estudios para la RPC de $M$. tuberculosis en LCR $\mathrm{LC}^{3,6,23}$. Este hallazgo pudiese explicarse por la gran antigenicidad de $M$. tuberculosis que resulta en la producción de anticuerpos detectables mediante ELISA pese a que la carga misma de antígenos micobacterianos sea muy baja en la muestra de $\mathrm{LCR}^{8,24}$.

El test de ADA en LCR entregó dos resultados falsamente negativos, en casos que sí fueron detectados correctamente por IgM anti-A60. Estos casos correspondieron a MTBC confirmada por RPC, no por cultivo, lo que pudiese reflejar una muy baja carga micobacteriana en la muestra de LCR, o menos probablemente, corresponder a resultados falsamente positivos de la RPC para M. tuberculosis.

En el presente estudio, IgM anti-A60 detectó correctamente una mayor proporción de casos de MTBC que las otras clases de inmunoglobulinas. En la tuberculosis pulmonar post primaria, se han descrito títulos altos de IgG y títulos bajos de IgM en plasma, mientras que títulos elevados de IgM sugerirían reactivación de una infección antigua $^{16,25}$. En MTBC, la mayoría de los estudios publicados ha incluido sólo IgG anti-A60. Pocos reportes existen sobre las tres clases de anticuerpos. De éstos, dos encontraron un rendimiento global bajo para $\operatorname{IgM}$ en LCR en relación a $\operatorname{IgG}$ e $\operatorname{IgA}{ }^{18,20}$, mientras que un tercer estudio encontró que en suero y en LCR la positividad de IgM anti-A60 era mayor que la de IgG en MTBC y en tuberculosis espinal ${ }^{19}$. Diferentes estadios de la infección tuberculosa podrían en parte explicar estas discrepancias.
La especificidad de la IgM anti-A60 no resultó superior a la de ADA, encontrándose falsos positivos en un número pequeño de muestras del grupo control. Reportes de inmunodiagnóstico de alta especificidad mediante anti-A60 han sido descritos exclusivamente en ausencia de infecciones en grupo control ${ }^{12}$. Los anticuerpos anti-antígeno A60 no son especie-específico y pueden ser detectados en otras infecciones por micobacterias atípicas y en lepra, habiéndose también reportado reacciones cruzadas con Corynebacteria y Nocardia $\mathrm{sp}^{16}$.

Valores de ADA y de anti-A60 elevados se concentraron particularmente en los casos de meningitis criptocóccica del grupo control. No existe evidencia de que Cryptococcus sp. pueda compartir antígenos con $M$. tuberculosis; sin embargo, algunos autores han sugerido que pudiese existir respuesta inmunológica compartida. Este hecho pudiese también representar infección tuberculosa antigua, efecto de vacunación BCG o uso de test cutáneo de tuberculina (PPD) y persistencia de anticuerpos medibles ${ }^{26,27}$. La meningitis criptocóccica puede ser rápidamente descartada mediante tinción con tinta China, con una alta sensibilidad y especificidad en relación al estándar de oro (cultivo de hongos). Por lo tanto, en la práctica clínica sería recomendable excluir meningitis criptocóccica en todos los casos de sospecha de MTBC, de manera a incrementar la especificidad tanto de ADA como de anti-A60.

En el presente estudio se excluyó la meningitis bacteriana aguda del grupo control debido a que en la práctica clínica estos casos son rápidamente diagnosticados y son más bien las meningitis a líquido claro y las meningitis crónicas las que pueden compartir con MTBC la forma de presentación clínica y alteraciones de LCR, complicando el diagnóstico. Sin embargo, es importante recordar que otras situaciones clínicas como la meningitis bacteriana aguda parcialmente tratada, que no fue incluida en este estudio, pueden constituir parte del diagnóstico diferencial de la $\mathrm{MTBC}^{28}$.

Como principal limitante del presente estudio no se incluyeron pacientes cursando tuberculosis en otras localizaciones (extra-meníngeas) ni se tuvo acceso a historia clínica que permitiese evaluar el antecedente de infección tuberculosa pasada, vacunación BCG o la presencia de 
co-infección con VIH. Se desconoce si los anticuerpos anti-A60 en LCR pudiesen variar en esas circunstancias. No está claro tampoco en qué momento de la infección meníngea las inmunoglobulinas anti-A60 se hacen detectables en LCR ni cuánto tiempo persisten luego de que la MTBC es tratada. La capa lipídica de la micobacteria que protege las glicoproteínas de la pared celular puede ser lisada bajo la acción de fármacos antituberculosos, liberando nuevos epítopes que son nuevos estímulos inmunológicos. Esto ha sido mostrado en estudios en que bajo tratamiento antituberculoso algunos pacientes presentan inicialmente un aumento de los anticuerpos monoclonales u oligoclonales contra nuevos antígenos ${ }^{26,29}$. Por otra parte, en la tuberculosis pulmonar estudios han mostrado que bajo terapia antituberculosa los niveles de IgG séricos disminuyen ${ }^{16}$. Se desconoce la situación específica de la duración y persistencia de anticuerpos anti-A60 bajo tratamiento, lo cual pudiese ser de interés en lugares donde el tratamiento antituberculoso se inicia en forma empírica, antes de tener confirmación diagnóstica.

En conclusión, los resultados del presente estudio sugieren que IgM anti-A60 en LCR pudiese servir de método diagnóstico complementario en el diagnóstico de la MTBC. Dado que no existe un método único que sea sensible y especifico, la combinación de diferentes pruebas puede ser necesaria para configurar correctamente este diagnóstico. Estudios prospectivos y a mayor escala serían necesarios para confirmar estos hallazgos.

\section{Resumen}

Antecedentes: El diagnóstico de meningitis tuberculosa (MTBC) se ve limitado por la ausencia de técnicas diagnósticas rápidas y precisas en líquido cefalorraquídeo (LCR). En este estudio evaluamos la respuesta inmunológica de anticuerpos anti-antígeno A60 de Mycobacterium tuberculosis en LCR en comparación a la determinación de adenosina deaminasa (ADA). Métodos: Un total de 63 muestras de LCR fueron estudiadas mediante ELISA indirecto para detección de IgG, IgM e IgA anti-A60. Estas muestras incluyeron 17 casos de MTBC confirmada y 46 controles con otras infecciones. Resultados: Los títulos de IgG, IgM e IgA anti A-60 resultaron significativamente superiores en casos de MTBC versus controles ( $p>0,01)$. $\mathrm{El}$ anticuerpo con mejor poder discriminatorio resultó IgM, con un área bajo la curva ROC de 0,928 (95\% IC 0,834$0,978)$, comparado a 0,863 (95\% IC: $0,752-0,936)$ para $\operatorname{ADA}(\mathrm{p}=\mathrm{NS})$. La sensibilidad de IgM anti-A60 (nivel de corte $>0,06 \mathrm{U} / \mathrm{ml}$ ) fue de $94,1 \%$ versus $88,2 \%$ para ADA (nivel de corte $>6,2 \mathrm{U} / \mathrm{ml}$ ), $\mathrm{p}=\mathrm{NS}$. Ambos IgM anti-A60 y ADA presentaron la misma especificidad baja-moderada (80,4\%). Dos casos de MTBC fueron correctamente identificados por IgM anti-A60 pero no por ADA. Conclusión: La detección de anticuerpos anti-A60 (IgM) puede ser de ayuda en el diagnostico de MTBC en forma complementaria a la determinación de ADA. La baja especificidad de ambos tests constituye su principal limitante.

\section{Referencias bibliográficas}

1.- Berenguer J, Moreno S, Laguna T. Tuberculous meningitis in patients infected with human immunodeficiency virus. N Engl J Med 1992; 326: 668-72.

2.- $\quad$ Fitzgerald D. H.D.W. Mycobacterium tuberculosis. In: Mandell, Douglas and Bennett's Principles and Practice of Infectious Diseases. Mandell GL, Bennett JE, Dolin R, eds. $6^{\text {th }}$ ed. 2010. Elsevier, Churchill Livingstone, Philadelphia, Pa, USA., pp 2852-85.

3.- Christie L J, Loeffler A M, Honarmand S, Flood J M, Baxter R, Jacobson S, et al. Diagnostic challenges of central nervous system tuberculosis. Emerg Infect Dis 2008; 14: 1473-5.

4.- Porkert M T, Sotir M, Parrott-Moore P, Blumberg H M. Tuberculous meningitis at a large inner-city medical center. Am J Med Sci 1997; 313: 325-31.

5.- Choi S H, Kim Y S, Bae I G, Chung J W, Lee M S, Kang J M, et al. The possible role of cerebrospinal fluid adenosine deaminase activity in the diagnosis of tuberculous meningitis in adults. Clin Neurol Neurosurg 2002; 104: 10-5.
6.- Pai M, Flores L L, Pai N, Hubbard A, Riley L W, Colford J M Jr. Diagnostic accuracy of nucleic acid amplification tests for tuberculous meningitis: a systematic review and meta-analysis. Lancet Infect Dis 2003; 3 : 633-43.

7.- Kashyap R S, Dobos K M, Belisle J T, Purohit H J, Chandak N H, Taori G M, et al. Demonstration of components of antigen 85 complex in cerebrospinal fluid of tuberculous meningitis patients. Clin Diagn Lab Immunol 2005; $12: 752-8$.

8.- Alifano M De Pascalis R, Sofia M, Faraone S, Del Pezzo M, Covelli I. Detection of IgG and IgA against the mycobacterial antigen A60 in patients with extrapulmonary tuberculosis. Thorax 1998; 53: 377-80.

9.- Daniel T M, Debanne S M. The serodiagnosis of tuberculosis and other mycobacterial diseases by enzyme-linked immunosorbent assay. Am Rev Respir Dis 1987; 135: 1137-51.

10.- Grange J M. The humoral immune response in tuberculosis: its nature, biological role and diagnostic usefulness. Adv Tuberc Res 1984; 21: 1-78.

11.- Van Vooren J P, Farber C M, Motte S, De Bruyn J, Legros F, Yernault J C. Assay of specific antibody response to mycobacterial antigen for the diagnosis of a pleural effusion in a patient with AIDS. Tubercle 1988; 69: 303-5.

12.- Chandramuki A, Lyashchenko K, Kumari H B, Khanna N, Brusasca H, Gourie-Devi M, et al. Detection of antibody to Mycobacterium tuberculosis protein antigens in the cerebrospinal fluid of patients with tuberculous meningitis. J Infect Dis 2002; 186: 678-83.

13.- Miorner H, Sjobring U, Nayak P, Chandramuki A. Diagnosis of tuberculous meningitis: a comparative analysis of 3 immunoassays, an immune complex assay and the polymerase chain reaction. Tuber Lung Dis 1995; 76: 381-6.

14.- Chandramuki A, Bothamley G H, Brennan P J, Ivanyi J. Levels of antibody to defined antigens of Mycobacterium tuberculosis in tuberculous meningitis. J Clin Microbiol 1989; 27: 821-5

15.- Kashyap R S, Ramteke S P, Deshpande P S, Purohit H J, Taori G M, Daginawala H F. Comparison of an adenosine deaminase assay with ELISA for the diagnosis of tuberculous meningitis infection. Med Sci Monit 2007; 13: BR 200-4. 
16.- Verma R J, Jain A. Antibodies to mycobacterial antigens for diagnosis of tuberculosis. FEMS Immunol Med Microbiol 2007; 51: 453-61.

17.- Sindic C J M, Boucquey D, Van Antwerpen M P, Baelden M C, Laterre C, Cocito C. Intrathecal synthesis of anti-mycobacterial antibodies in patients with tuberculous meningitis. An immunobloting study. J Neurol Neurosurg Psychiatry 1990; 53: 662-6.

18.- Maheshwari A, Gupta H L, Gupta S, Bathia R, Datta K K. Diagnostic utility of estimation of mycobacterial antigen A60 specific immunoglobulins in serum and CSF in adult neurotuberculosis. J Commun Dis 2000; 32: 54-60.

19.- Ghoshal U, Kishore J, Kumar B, Ayyagari A. Serodiagnosis of smear and culturenegative neurotuberculosis with enzyme linked immunosorbent assay for anti-A60 immunoglobulins. Indian J Pathol Microbiol 2003; 46: 530-4.

20.- López-Cortés L F, Nogales-Pérez M C, Gómez-Mateos J, Jiménez-Hernández D, Jiménez-Mejias E, Pachón-Díaz J. Antibodies to antigen A60 in cerebrospinal fluid from patients with tuberculous meningitis. Eur J Clin Microbiol Infect Dis 1994; 13: 490-5.

21.- Zou Y L, Van Antwerpen M P, Shi G Q, Chen Q X, Sindic C J M, Cocito C. Analysis of tuberculous meningitis cases by immunoblotting assay based on a mycobacterial antigen complex. Clin Diagn Lab Immunol 1994; 1: 353-6.

22.- Garg R K. Tuberculosis of the central nervous system. Postgrad Med J 1999; 75: 133-40.

23.- Brienze V M, Tonon A P, Pereira F J, Liso E, Tognola W A, Dos Santos M A, et al. Low sensitivity of polymerase chain reaction for diagnosis of tuberculous meningitis in southeastern Brazil. Rev Soc Bras Med Trop 2001; 34: 389-93.

24.- Kinnman J, Fryden A, Eriksson S, Moller E, Link H. Tuberculous meningitis: immune reactions within the central nervous system. Scand J Immunol 1981; 13: 289-96.

25.- Gevaudan M J, Bollet C, Charpin D, Mallet M N, De Micco Ph. Serological response of tuberculosis patients to antigen 60 of BCG. Eur J Epidemiol 1992; 8: 666-76.

26.- Montoya J, Stanford J L. Comparison of antibody clones in cerebrospinal fluid of patients with tuberculous meningitis before and during chemotherapy using agarose gel isoelectric focusing with antigen immunoblotting. Phil J Microbiol Infect Dis. 1996; 25: 61-9.

27.- Bardana E J, McClatchy J K, Farr R S, Minden P. Universal occurrence of antibodies to tubercle bacilli in sera from non-tuberculous and tuberculous individuals. Clin Exp Immunol 1973; 13: 65-77.

28.- Kashyap R S, Kainthia R P, Satpute R M, Agarwal N P, Chandak N H, Purohit H J, et al. Differential diagnosis of tuberculous meningitis from partially-treated pyogenic meningitis by cell ELISA. BMC Neurol 2004; 4: 16-19

29.- Kaplan M H, Chase M W. Antibodies to mycobacteria in human tuberculosis: Development of antibodies before and after antimicrobial therapy. J Infect Dis 1980; 142: 825-34. 